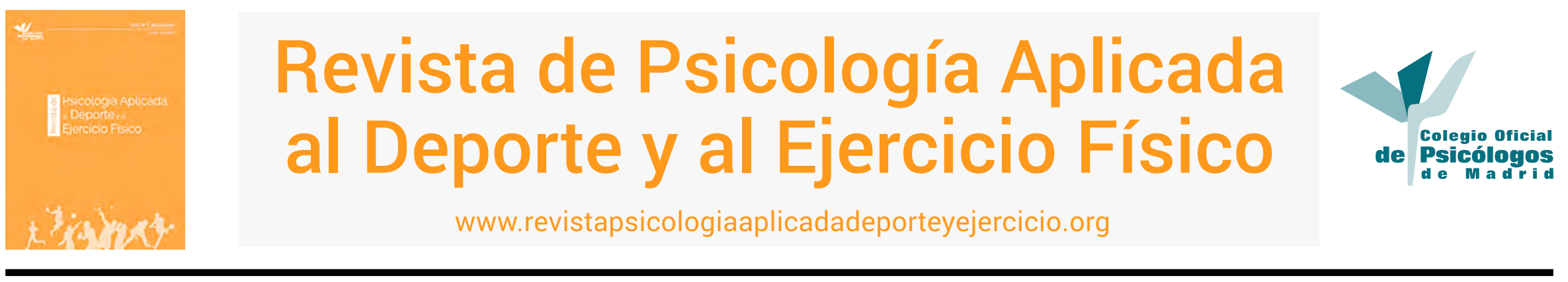

\title{
Motivación y toma de decisiones en voleibol: Influencia de los años de experiencia
}

\author{
Eva León Zarceño', Salvador Boix Vilella', Miguel Ángel Serrano Rosa ${ }^{2}$, Nerea Paredes López ${ }^{1}$ \\ ${ }^{1}$ Universidad Miguel Hernández de Elche, España, ${ }^{2}$ Universitat de València, España
}

RESUMEN: La motivación y la toma de decisiones se consideran factores influyentes en el rendimiento deportivo. El objetivo principal del presente trabajo fue describir los niveles motivacionales y la dimensión subjetiva de la toma de decisiones que registran jugadores de voleibol en función de los años de experiencia acumulados practicando dicho deporte. Para ello, se establecieron tres rangos a partir de sus años de práctica: menos de cinco años, entre cinco y diez años y más de diez años. La muestra estaba formada por 50 participantes (22 hombres y 28 mujeres) que tenían una edad media de 22 años ( $D T$ = 3.33) y competían en categoría sénior o juvenil. Los instrumentos utilizados fueron la Escala de Motivación Deportiva (EMD) y el Cuestionario de Estilo de Decisión en el Deporte (CETD). Los resultados significativos encontrados revelan que los jugadores más expertos registraban los mayores niveles de motivación extrínseca guiada por las recompensas externas y de motivación intrínseca hacia la estimulación con respecto al grupo con menos años de experiencia. En el caso de la toma de decisiones, los niveles de ansiedad y agobio al decidir eran significativamente mayores entre los practicantes del grupo de cinco a diez años con respecto al grupo más experto. Además, se ha observado la existencia de relaciones entre variables motivacionales con la competencia decisional percibida y el compromiso con el aprendizaje decisional. Se concluye que la intervención psicológica debe ser prioritaria con los jugadores que acumulan una práctica de voleibol inferior a cinco años y de cinco a diez años para mejorar su bienestar y rendimiento deportivo.

PALABRAS CLAVES: Motivación, toma de decisiones, voleibol, deportes colectivos.

\section{Motivation and Decision Making in Volleyball: Influence of Years of Experience}

\begin{abstract}
Motivation and decision-making are considered influential factors in sports performance. The main objective of this study was to describe the motivational levels and the subjective dimension of the decision-making found in volleyball players as a function of the years of experience practicing that sport. For this purpose, three groups were established based on their years of practice: less than five years, between five and ten years, and more than ten years. The sample consisted of 50 participants (22 men and 28 women) with a mean age of 22 years (SD = 3.33) and competing in the senior or junior categories. The instruments used were the Sports Motivation Scale (SMS) and the Sport Decision Style Questionnaire (SDSQ). The significant results found show that the most experienced players had the highest levels of extrinsic motivation, which favors not self-determined behavior guided by external rewards. This group also showed higher levels in the intrinsic motivation toward stimulation variable. As regards decision-making, levels of anxiety and overwhelm when deciding were significantly greater among those five to ten years of sports practice as compared to the more expert group. In addition, relations have been seen to exist between motivational variables and the perceived decisional competence and the commitment to decisional learning. It is concluded that psychological intervention should be a priority with players who accumulate a volleyball practice of less than five years and from five to ten years to improve their well-being and sports performance.
\end{abstract}

KEYWORDS: Motivation, decision-making, volleyball, collective sports.

\section{Motivação e tomada de decisão no voleibol: Influência de anos de experiência}

RESUMO: Motivação e tomada de decisão consideram-se fatores influentes no desempenho atlético. O principal objetivo deste estudo foi descrever os níveis motivacionais e a dimensão subjetiva da tomada de decisões que registam os jogadores de vôlei, dependendo

Eva León, Salbador Boix y Nerea Paredes son psicólogas y pertenecen al Departamento de Psicología de la Salud de la Universidad Miguel Hernández.

Miguel Ángel Serrano es psicólogo y pertenece al Departamento de Psicobiología de la Universitat de València.

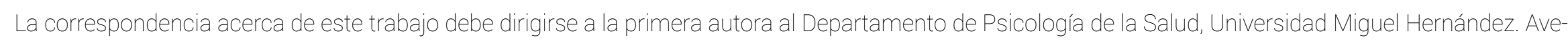
nida de la Universidad, s/n. 03202. Elche (España). E-mail:

(cc) $\mathbf{E r}$-No-nd Este es un artículo Open Access bajo la licencia 
dos anos de experiência acumulada na prática do esporte. Foram estabelecidas tres faixas em função seus anos de prática: menos de cinco anos, cinco a dez anos e mais de dez anos. A amostra foi composta por 50 participantes (22 homens e 28 mulheres) que tinham uma idade média de 22 anos ( $\mathrm{DP}=3,33$ ) e competiam na categoria sênior ou júnior. Os instrumentos utilizados foram a Escala Motivação Desportiva (EMD) e Questionàrio de Estilo de Decisão no Desporto (QETD). Os resultados significativos encontrados revelam que os jogadores mais experientes registavan os mais altos níveis de motivação extrínseca guiada pelas gratificações externas e de motivação intrínseca para a estimulação com respeito ao grupo com menos anos de experiência. No caso de tomada de decisão, os níveis de ansiedade e angústia ao decidir foram significativamente maiores entre os praticantes do grupo de cinco a dez anos em comparação com o grupo com mais experiência. Além disso, observou-se a existência de relações entre as variáveis motivacionais com a competência decisória percebida e o compromisso com a aprendizagem decisória. Conclui-se que a intervenção psicológica deve ser uma prioridade com os jogadores que acumulam uma prática de voleibol de menos de cinco anos e de cinco a dez anos para melhorar seu bem-estar e desempenho desportivo.

PALAVRAS-CHAVE: Motivação, tomada de decisão, voleibol, esportes de equipe.

Artículo recibido: 05/04/2017 | Artículo aceptado: 06/06/2017

El deporte de competición tiene como objetivo la expresión o la mejora de la condición física o psíquica, el desarrollo de las relaciones sociales y la obtención de resultados en competición oficial (Unisport, 1992). En este contexto, las personas se interesan por aprender gestos técnicos, tácticas y estrategias para poder competir en las mejores condiciones (Estrada-Contreras y Pérez-Córdoba, 2015). El voleibol es un deporte colectivo de estrategia en el que existe una gran incertidumbre y donde la toma de decisiones más apropiada en cada momento es fundamental para alcanzar resultados positivos en la competición (Gil-Arias, Moreno, Claver, Moreno y Del Villar, 2016). Las singularidades que rodean la práctica de voleibol, como por ejemplo las continuas transiciones entre ataque y defensa o el rápido ritmo del juego, ofrecen a los investigadores una oportunidad excelente para el análisis de variables como la toma de decisiones y/o la motivación (Gil-Arias et al., 2010; Johnson, 2006). De hecho, hay autores que las han relacionado y resaltan la influencia de las orientaciones motivacionales sobre el proceso de toma de decisiones (Zeelenberg, Nelissen y Pieters, 2008). Estas conexiones señalan la conveniencia de vincular los procesos cognitivos y motivacionales en etapas de formación (Jiménez y López-Zafra, 2009). En el caso concreto de la toma de decisiones, el reciente trabajo de Conejero, Claver, Fernández-Echevarría, González-Silva y Moreno (2017), centrado en la acción de recepción en voleibol, ha desarrollado y validado un instrumento de observación que permite evaluar la toma de decisiones en etapas de formación. A pesar de éste y otros estudios, se ha considerado insuficiente la relevancia práctica de la toma de decisiones dentro del campo de la Psicología del Deporte (García, Ruiz y Graupera, 2009) por lo que parece evidente la necesidad de nuevos trabajos.

Las habilidades tácticas, necesarias para la competición, se definen como el conocimiento sobre las adaptaciones en el juego y la toma de decisiones en el campo (Thomas, French y Humphries, 1986). En el caso de la toma de decisiones, su concepto hace referencia al proceso por el cual el deportista decide cómo actuar o reaccionar en función de las demandas del entorno para conseguir distintos objetivos de rendimiento (Hodges, Huys y Starkes, 2007). Pese a que la toma de decisiones tradicionalmente se ha analizado como una habilidad cognitiva, el modelo de estilos de toma de decisión en el deporte de Ruiz, Graupera y Navarro (2000) centra su análisis en los aspectos emocionales y motivacionales del deportista a la hora de tomar decisiones en contextos de entrenamiento y competición. Según Konzag (1992) los procesos decisionales en el deporte se engloban en tres fases diferentes: (1) la preparación de la decisión centrada en el análisis de la situación y de las posibilidades de acción; (2) el acto de decisión donde se elige el gesto técnico-táctico a realizar; (3) la realización y control de la decisión. Autores como Chamberlain y Coelho (1993), que estudian el perfil decisional, centran sus trabajos en valorar el grado de autoconfianza ante la toma de decisiones y llegan a la conclusión que el aumento de la autoconfianza es uno de los aspectos que caracteriza las diferencias entre expertos y novatos en el deporte. Otros autores han señalado que la percepción de competencia decisional muestra un considerable incremento lineal conforme los deportistas van mejorando su nivel deportivo (Ruiz et al., 2002).

La motivación en el deporte es una variable que ha estado presente en numerosas investigaciones como así se observa en el reciente trabajo de revisión de Clancy, Herring, Maclntyre y Campbell (2016). Dicha importancia se debe a que sus componentes intrapersonales e interpersonales tienen importantes implicaciones en las prácticas de entrenamiento de deportistas (Irwin y Feltz, 2016). Además, la motivación deportiva es un elemento clave para lograr el compromiso y 
la adherencia al deporte, ya que es el más importante e inmediato determinante del comportamiento humano (Iso-Ahola y St.Clair, 2000), pues lo despierta, le da energía, lo dirige y lo regula (Roberts, 2001). De manera general, la motivación es un concepto que se usa cuando se quieren describir las fuerzas que actúan sobre, o dentro de, un organismo, para iniciar y dirigir la conducta de éste (Palmero, 2005).

El estudio de la motivación viene siendo aplicado en diversos ámbitos de la vida cotidiana y de la conducta, siendo uno de ellos el de la actividad física y el deporte (Moreno y Martínez, 2006). La teoría de la autodeterminación analiza el grado en que las conductas humanas son volitivas o autodeterminadas (Deci y Ryan, 1985), es decir, el grado en que las personas realizan sus acciones de forma voluntaria, por propia elección (Carratalá, 2004). La motivación autodeterminada se corresponde con la motivación intrínseca, mientras que la motivación extrínseca favorece la conducta no autodeterminada e incluso caracterizada por la ausencia de motivación (Standage y Vallerand, 2014). La motivación extrínseca está determinada por recompensas externas y la motivación intrínseca implica voluntariedad y disfrute (Catalá, Peñacoba, Velasco, Jareño y Fernández-Serrano, 2016). Por ejemplo, un deportista estará intrínsecamente motivado cuando realice su deporte por la satisfacción y las emociones positivas que derivan de la práctica del mismo. En el caso de los deportistas extrínsecamente motivados, otorgarán gran importancia a las consecuencias externas derivadas de la realización de las actividades dentro de su práctica deportiva, aunque no intervengan directamente en la acción (Deci y Ryan, 1985). Asimismo, los deportistas que no obtengan refuerzos ni intrínseca ni extrínsecamente, no se encontrarán motivados para realizar el deporte en cuestión (Ryan y Deci, 2000) y aumentará el riesgo de abandono de la práctica deportiva (Jõesaar, Hein y Hagger, 2011).

En función de los antecedentes expuestos, se propone como objetivo para el presente trabajo: describir los niveles motivacionales y la dimensión subjetiva de la toma de decisiones que registran jugadores de voleibol en función de los años de experiencia practicando dicho deporte, indagando acerca de las posibles relaciones existentes entre ambas variables. La primera hipótesis planteada considera que los jugadores más expertos serán los que presenten menores niveles de motivación, en la línea del trabajo de Reche, Cepero y Rojas (2010), y que ésta sea más extrínseca (Baena-Extremera y Granero-Gallegos, 2015). La segunda hipótesis contempla que los jugadores más expertos obtendrán mejores puntuaciones en las subescalas relacionadas con la toma de decisiones ya que existe un incremento lineal de la competencia decisional conforme los deportistas van mejorando su nivel deportivo (García et al., 2009). Para finalizar, la tercera hipótesis contempla la existencia de relaciones entre variables motivacionales y variables decisionales como se recoge en el trabajo de Zeelenberg et al. (2008).

\section{Método}

\section{Participantes}

La muestra estuvo formada por un total de 50 participantes con una edad media de 22 años $(D T=3.33)$. Las 28 mujeres (56\%) y los 22 hombres (44\%) fueron agrupados en función de sus años de experiencia practicando voleibol. En este caso, 11 deportistas (22\%) acumulaban una práctica menor a cinco años, 13 deportistas (26\%) practicaban entre cinco y diez años y los 26 restantes (52\%) acumulaban más de diez años. El grupo con menos años de experiencia registraba una edad media de 20.18 años ( $D T=2.18)$, el grupo con una práctica intermedia obtenía una media de 21.54 años $(D T=2.79)$ y el grupo más experto registraba una edad media de 23.00 años $(D T=3.67)$. El criterio de agrupación de los participantes se basa en el empleado en los trabajo de Reche et al. (2010) y Boix (2016) donde se clasifica a los deportistas con una experiencia deportiva mayor o menor a cinco años. Además, en el presente trabajo se incluye un tercer grupo formado por participantes con una alta experiencia (igual o superior a diez años), criterio de agrupación empleado en el trabajo de García-Naveira y Ruiz-Barquín (2013). Por último, los integrantes de la muestra se ejercitaban cuatro días a la semana, en sesiones de 90 minutos y competían en la primera o segunda división autonómica valenciana. La participación fue voluntaria.

Se establecieron tres criterios de inclusión para que los participantes pudieran formar parte de la muestra. El primero de ellos tenía que ver con la edad mínima que fue establecida en 18 años. El segundo criterio precisaba la tenencia en vigor de la correspondiente licencia dentro de la federación valenciana de voleibol. El tercer y último criterio recogía la obligatoriedad de pertenecer a alguno de los equipos masculinos o femeninos seleccionados para participar en el estudio. Respecto a los criterios de exclusión, se establece la no participación de aquellos jugadores y jugadoras que pese a tener licencia federativa y tener la mayoría de edad están en tratamiento de lesiones que les imposibilitan competir en sus respectivos equipos.

\section{Instrumentos}

Cuestionario sociodemográfico ad hoc. Previa a la cumplimentación de los cuestionarios de motivación y toma de 
decisiones se administró un breve cuestionario sociodemográfico, de elaboración propia. En éste cuestionario se recogían algunos hábitos físico-deportivos y datos personales considerados relevantes.

Escala de Motivación Deportiva (EMD). Para evaluar la motivación de los deportistas se utilizó la versión española de Balaguer, Castillo y Duda (2007) de la Sport Motivation Scale (SMS-28) de Pelletier et al. (1995). La escala consta de 28 ítems divididos en siete subescalas: (1) motivación intrínseca para experimentar estimulación; (2) motivación intrínseca para conseguir cosas; (3) motivación intrínseca hacia el conocimiento; (4) regulación identificada; (5) regulación introyectada; (6) regulación externa; (7) amotivación. Las respuestas a cada ítem se representan a través de una escala de tipo Likert que oscila de uno a siete, donde uno significa que la expresión presentada no tiene nada que ver conmigo y siete que se ajusta totalmente a mí. Investigaciones previas han confirmado la fiabilidad del instrumento en muestras españolas (Balaguer, Castillo y Duda, 2003, 2007). En el caso del trabajo de Núñez, Martín-Albo y Navarro (2007) se encuentran unos alfa de Cronbach que oscilan entre los .73 de la subescala regulación introyectada y los 79 de las subescalas regulación externa y motivación intrínseca hacia el conocimiento. En el presente trabajo, seis de las siete subescalas obtienen unos índices de fiabilidad alfa de Cronbach superiores a .74 con la salvedad de la subescala amotivación que registra una puntuación de 67.

Cuestionario de Estilo de Decisión en el Deporte (CETD). Este instrumento fue desarrollado y validado por Ruiz y Graupera (2005). Consta de 30 ítems y las respuestas a cada uno se representan a través de una escala de tipo Likert, que oscila entre uno y cuatro. En dicha escala, el uno representa el máximo desacuerdo con la afirmación y el cuatro, el acuerdo total. El instrumento mide tres escalas de diez ítems cada una: (1) competencia decisional percibida (percepción que la persona tiene sobre su propia competencia para decidir en su deporte, tanto por la opinión que otras personas importantes tengan sobre ella, como por su propia comparación con compañeros y contrincantes); (2) ansiedad y agobio al decidir (referida a las circunstancias que generan estrés, y a miedos y temores del deportista relacionados con la toma de decisiones en el propio deporte); (3) compromiso en el aprendizaje decisional (se corresponde con el esfuerzo y la responsabilidad que el deportista muestra en la mejora de su competencia para decidir y la capacidad de seguir los consejos de sus entrenadores para conseguirlo). El cuestionario ha sido validado en deportistas españoles de dife- rentes modalidades deportivas y con diferentes niveles de pericia (Ruiz y Graupera, 2005). En el trabajo de García et al. (2009) se obtienen unos valores alfa de Cronbach de .81 para la escala de competencia decisional percibida, de .82 para la escala de ansiedad y agobio al decidir y de .73 para la escala de compromiso en el aprendizaje decisional. En el presente trabajo, las escalas competencia decisional percibida y ansiedad y agobio al decidir registran un índice de fiabilidad alfa de Cronbach de .91 y la escala compromiso con el aprendizaje obtiene .70 .

\section{Procedimiento}

Para la realización del estudio se contactó con los tres entrenadores responsables de la coordinación de los diferentes equipos universitarios y clubes participantes (equipo masculino y femenino de una universidad pública de la Comunidad Valenciana y equipos externos a la universidad de la misma localidad). Posteriormente, se realizaron breves reuniones explicativas con los responsables para poner en conocimiento los objetivos del estudio y el procedimiento del mismo. Todos ellos accedieron a participar voluntariamente en la investigación. A continuación, se informó a los jugadores de la posibilidad de participar en el estudio recordándoles que dicha participación era totalmente voluntaria y que todas sus respuestas serían tratadas de manera confidencial, como así se recoge en la Declaración de Helsinki (1964), aprobada en Fortaleza (2013). Todos ellos aceptaron participar. Para ello debían firmar un consentimiento informado y disponían de 20 minutos aproximadamente para la cumplimentación de las escalas. Los cuestionarios se cumplimentaron de manera grupal antes del entrenamiento estando presente un miembro del equipo de investigación durante la cumplimentación de los cuestionarios atendiendo cualquier posible pregunta o duda de los participantes.

\section{Análisis de datos}

Los datos recogidos se analizaron mediante el programa estadístico SPSS en su versión 20.0. En primer lugar, se eliminaron los outliers y se comprobó que las variables evaluadas cumplían el supuesto de normalidad estadística. A continuación, se llevaron a cabo los análisis descriptivos y de frecuencias. Finalmente, se utilizó el modelo lineal general univariante (ANOVAs de una vía) para la comparación de los grupos, utilizando las comparaciones múltiples de Bonferroni como pruebas post-hoc. El nivel de significación estadística fue establecido en $p<.05$. 


\section{Diseño de investigación}

El diseño de investigación en el presente estudio es empírico, no experimental, con una estrategia descriptiva-comparativa (Ato, López y Benavente, 2013). Se pretende, por tanto, describir las puntuaciones obtenidas en los diferentes instrumentos, sin ningún tipo de manipulación de las variables. Además, se van a comparar los resultados obtenidos por los tres grupos de participantes establecidos en función de sus años practicando voleibol. Por último, el estudio cuenta con una única medición por lo que se considera de corte transversal.

\section{Resultados}

En relación a los niveles de motivación, el efecto principal de la variable motivación intrínseca hacia la estimulación resulta estadísticamente significativo $(F 2,47=3.93, p<.026$ $\eta 2 p=.143$, power $=.679)$. Al resultar significativo, se llevan a cabo las pruebas post hoc Bonferroni confirmando que el grupo con una práctica inferior a cinco años de experiencia en voleibol obtiene menor motivación hacia la estimulación frente al grupo de más de diez años $(p=.023)$. Respecto a la motivación extrínseca identificada el efecto principal resulta nuevamente significativo $(F 2,47=3.66, p<.033 \eta 2 p=.135$, power $=.647$ ). Los análisis post hoc Bonferroni confirman que las diferencias se dan entre el grupo de menos de cinco años de práctica y el grupo que lleva más de diez años practicando voleibol ( $p=0.028)$, siendo la motivación identificada mayor en el grupo más experto. En el caso de la motivación extrínseca introyectada se detectan nuevas diferencias significativas ( $F 2,47=4.46, p<.017 \eta 2 p=.160$, power $=.738$ ). Los post hoc señalan diferencias entre el grupo con menos de cinco años de práctica frente al de más de diez años ( $p=$ .027) y entre el grupo de menos de cinco años con respecto al grupo de cinco a diez años de práctica $(p=.033)$. En ambos casos, el grupo con una práctica inferior a cinco años es el que obtiene los menores niveles de motivación introyectada. Por último, el efecto principal de la motivación extrínseca de regulación externa resulta significativo (F2,47 = 4.95, $p<$ $.011 \eta 2 p=.174$, power $=.784)$. En este caso, las diferencias significativas halladas se vuelven a dar entre el grupo con una práctica inferior a cinco años con respecto al grupo de más de diez años $(p=.009)$, obteniéndose menores niveles de motivación extrínseca de regulación externa en el grupo más inexperto. La Tabla 1 muestra los resultados descriptivos obtenidos para las siete subescalas de motivación y concreta dónde se encuentran las diferencias significativas encontradas entre grupos.

En relación a la dimensión subjetiva de la toma de decisiones, el efecto principal es significativo en la subescala ansiedad y agobio al decidir $(F 2,47=3.64, p<.034 \eta 2 p=.134$,

\section{Tabla 1. Estadísticos descriptivos, desviaciones típicas y diferencias significativas entre grupos}

\begin{tabular}{|c|c|c|c|c|}
\hline & $\begin{array}{l}\text { Menos } 5 \text { años } \\
\text { Media (DT) }\end{array}$ & $\begin{array}{l}\text { 5-10 años } \\
\text { Media (DT) }\end{array}$ & $\begin{array}{l}\text { Más } 10 \text { años } \\
\text { Media (DT) }\end{array}$ & $\mathbf{p}$ \\
\hline \multicolumn{5}{|l|}{ (CETD) } \\
\hline Competencia decisional percibida & $1.90(0.64)$ & $1.95(0.62)$ & $2.29(0.72)$ & - \\
\hline Ansiedad y agobio al decidir & $2.26(0.64)$ & $2.74(0.89)$ & $2.02(0.78)$ & 5-10 años > Más 10 años* \\
\hline Compromiso con el aprendizaje decisional & $3.35(0.34)$ & $3.30(0.35)$ & $3.23(0.40)$ & - \\
\hline \multicolumn{5}{|l|}{$(E M D)$} \\
\hline Amotivación & $2.38(0.67)$ & $2.37(1.21)$ & $2.09(0.77)$ & - \\
\hline Motivación intrínseca hacia el conocimiento & $5.81(0.96)$ & $6.17(0.65)$ & $5.69(1.13)$ & - \\
\hline Motivación intrínseca hacia la estimulación & $5.57(1.11)$ & $6.06(0.88)$ & $6.36(0.54)$ & Menos 5 años < Más 10 años* \\
\hline Motivación intrínseca hacia la autosuperación & $5.82(1.04)$ & $6.00(0.74)$ & $5.97(0.91)$ & - \\
\hline Motivación extrínseca identificada & $4.43(1.70)$ & $5.23(1.05)$ & $5.55(0.89)$ & Menos 5 años < Más 10 años* \\
\hline Motivación extrínseca de regulación externa & $2.48(0.96)$ & $3.32(1.30)$ & $3.68(0.97)$ & Menos 5 años < Más 10 años* \\
\hline \multirow[t]{2}{*}{ Motivación extrínseca introyectada } & $3.45(1.64)$ & $4.94(1.33)$ & $4.80(1.27)$ & Menos 5 años < Más 10 años* \\
\hline & & & & Menos 5 años $<5-10$ años* \\
\hline
\end{tabular}

Nota: ${ }^{*} p<.05$ 
power $=.644$ ). Justo después, los análisis post hoc encuentran diferencias entre el grupo de cinco a diez años de práctica de voleibol con respecto al grupo de más de 10 años ( $p$ $=.029$ ). Los valores de ansiedad y agobio al decidir son más bajos en el grupo más experto. Véase Tabla 1.

A continuación, se selecciona la escala de motivación deportiva y el cuestionario de estilo de decisión en el deporte, con sus correspondientes subescalas, y se llevan a cabo los análisis de correlaciones de Spearman. Respecto a la primera subescala, competencia decisional percibida, se encuentran relaciones significativas de signo positivo en el nivel $p<.01$ con las variables: motivación intrínseca hacia la estimulación $(r=.40)$, motivación extrínseca hacia la regulación externa $(r=.56)$ y motivación extrínseca hacia la regulación introyectada $(r=.40)$. La subescala compromiso con el aprendizaje decisional también se relaciona de manera positiva, en el nivel $p<.01$, con las variables: motivación intrínseca hacia el conocimiento $(r=.55)$, motivación intrínseca hacia la autosuperación $(r=.44)$ y motivación externa de regulación introyectada $(r=.44)$. La Tabla 2 muestra las relaciones encontradas que se pueden considerar como bajas-medias. e introyectada). Además, el grupo más inexperto obtiene también menores puntuaciones en motivación extrínseca introyectada con respecto al grupo de práctica intermedia (aquellos deportistas con una experiencia de práctica en voleibol de entre cinco y diez años). Estos datos vienen a indicar que los deportistas más expertos buscan en la práctica de voleibol el desarrollo de aspectos tales como las relaciones sociales, un adecuado estado de forma o el prestigio que rodea al deportista. Según la Teoría de la Autodeterminación, el tipo de motivación que desarrollen los deportistas (motivación intrínseca, extrínseca o no motivación) va a depender en buena parte de la figura del entrenador (Deci y Ryan, 1985). Las puntuaciones obtenidas por los deportistas en las subescalas de motivación extrínseca en los diferentes grupos estudiados reflejan un aumento progresivo en este tipo de motivación. Así, conforme acumulan más años de experiencia practicando voleibol aumentan sus niveles de motivación extrínseca. Estos resultados coinciden con los obtenidos por Baena-Extremera y Granero-Gallegos (2015), con alumnos de educación física, donde se detecta que conforme avanza la edad, y por tanto los años de práctica, hay una motivación más extrínseca. El grupo

Tabla 2. Coeficientes de Correlación de Spearman entre las variables motivación y estilo decisional

\begin{tabular}{|c|c|c|c|c|c|c|c|c|c|c|}
\hline & 1 & 2 & 3 & 4 & 5 & 6 & 7 & 8 & 9 & 10 \\
\hline 1. Competencia decisional percibida & - & & & & & & & & & \\
\hline 2. Ansiedad y agobio al decidir & $-.357^{\star}$ & - & & & & & & & & \\
\hline 3. Compromiso con el aprendizaje decisional & $.314^{\star}$ & -.156 & - & & & & & & & \\
\hline 4. Amotivación & -.029 & -.013 & -.260 & - & & & & & & \\
\hline 5. MI hacia el conocimiento & .225 & .025 & $.553^{\star *}$ & -.252 & - & & & & & \\
\hline 6. MI hacia la estimulación & $.398 * \star$ & -.220 & .236 & -.163 & $.555^{\star \star}$ & - & & & & \\
\hline 7. MI hacia la autosuperación & .199 & -.182 & $.437 \star \star$ & .005 & $.777 \star \star$ & $.548 \star \star$ & - & & & \\
\hline 8. ME identificada & -.074 & -.038 & .033 & .170 & .114 & .251 & $.292^{\star}$ & - & & \\
\hline 9. ME regulación externa & $.558 * \star$ & -.096 & .205 & -.103 & $.311^{\star}$ & $.494^{\star *}$ & .261 & .257 & - & \\
\hline 10. ME regulación introyectada & $.400 \star *$ & -.160 & $.437 * *$ & -.120 & $.504^{\star *}$ & $.480 \star *$ & $.454^{\star \star}$ & .239 & $.594^{\star \star}$ & - \\
\hline
\end{tabular}

Nota: ${ }^{*} p<.05^{* \star} p<.01$

\section{Discusión}

Los deportistas principiantes, con una experiencia en voleibol inferior a cinco años, frente a los deportistas más expertos, con una práctica superior a diez años, presentan niveles de motivación inferiores en las tres variables que miden motivación extrínseca (identificada, regulación externa más experto sigue otorgando mayor valor, tras diez años de práctica, al sentimiento de estar inmerso en su deporte y a la emoción que sienten practicando voleibol. En definitiva, los resultados descriptivos apuntan que los jugadores más inexpertos del presente estudio presentan menores niveles motivacionales (intrínsecos y extrínsecos) por lo que se rechaza la primera hipótesis planteada, que esperaba mayo- 
res niveles motivacionales en el grupo con menos años de práctica (Reche et al., 2010).

Por otra parte, los resultados muestran que los participantes con una experiencia de práctica que oscila entre los cinco y diez años presentan mayor nivel de ansiedad y agobio al decidir. En el caso de los jugadores con una práctica superior a diez años obtienen menor ansiedad y agobio al decidir lo que viene a confirmar la segunda hipótesis planteada. Estos resultados van en la línea del trabajo de García et al. (2009) que considera que los perfiles decisionales de los jugadores y jugadoras de voleibol se van modificando según aumenta su nivel de pericia. El perfil óptimo de un deportista se da si obtiene una puntuación muy baja en la subescala ansiedad, moderada-alta en la subescala competencia decisional y muy alta en la subescala compromiso en el aprendizaje decisional, dando lugar un perfil en "V" (Ruiz y Graupera, 2005). Las diferencias entre grupos en ansiedad, podrían tener relación con el periodo de aprendizaje y con su nivel de pericia en la línea de otros trabajos previos (Jiménez, 2007, Ruiz et al., 2000). Destacar que al grupo con una práctica intermedia se le presuponen unos conocimientos específicos y por tanto podrían estar expuestos a mayores demandas y exigencias deportivas que influirían en su bienestar emocional.

Por último, se observan relaciones entre variables motivacionales y decisionales confirmando la tercera hipótesis del estudio. Estos resultados coinciden con los encontrados en el trabajo de Claver, Jiménez, Del Villar, García-Mas y Moreno (2015) que relaciona variables cognitivas con emocionales. La relación de la subescala competencia decisional percibida con las variables motivacionales es directa. Esto viene a señalar que una elevada percepción sobre la propia competencia se relaciona con elevados niveles de: estimulación hacia la práctica de voleibol, regulación externa y regulación introyectada. En relación a la subescala compromiso con el aprendizaje decisional, también se observa una relación directa. Por lo que, elevados niveles de esfuerzo en la mejora de su competencia para decidir se relacionan con elevados niveles de: disfrute por aprender cosas nuevas, autosuperación y regulación introyectada. En este sentido, es importante considerar prioritario el bienestar psicológico de los deportistas sin olvidar su rendimiento deportivo (Lorenzo, Gómez, Pujals y Lorenzo, 2012).

A modo de síntesis, se ha podido comprobar que los jugadores más expertos registran los mayores niveles de motivación extrínseca lo que viene a favorecer la conducta no autodeterminada que se guía por las recompensas externas. Este grupo también obtiene mayores puntuaciones en la variable motivación intrínseca hacia la estimulación por la práctica de voleibol con respecto al grupo más inex- perto. Los mayores niveles de ansiedad y agobio para decidir se dan en el grupo con una práctica que oscila entre los cinco y diez años. Por último, se ha observado la existencia de relaciones entre variables motivacionales con la competencia decisional percibida y el compromiso con el aprendizaje decisional.

Una de las limitaciones del presente estudio es el reducido número de participantes por lo que de cara a futuras investigaciones sería importante ampliar el tamaño muestral. Otra limitación es la ausencia de seguimiento de los participantes ya que el estudio sólo cuenta con una única medición. Los futuros trabajos deberían proyectar estudios longitudinales que posibilitaran la implantación de programas de intervención que tuvieran en cuenta las variaciones en los niveles motivacionales y de toma de decisiones de los jugadores en función de sus años de práctica. Entre las posibilidades, se cree interesante valorar la influencia del entrenador en los niveles motivacionales de sus jugadores ya que se considera un factor importante a tener en cuenta. También se podrían llevar a cabo estudios interculturales estableciendo diferencias de género y tratando de controlar posibles variables contaminadoras como la experiencia deportiva previa a la evaluación. Estos análisis se podrían llevar a cabo en otros deportes e incluso se podrían examinar las posibles diferencias entre deportes individuales vs. deportes colectivos. Por último, sería recomendable ampliar la participación a jugadores pertenecientes a clubes de otros municipios.

\section{Aplicaciones prácticas}

Los resultados encontrados ofrecen información relevante para diseñar programas de intervención psicológica aplicados de manera específica a las necesidades detectadas según los años de experiencia practicando voleibol. En primer lugar, los autores del presente trabajo consideran que cualquier estrategia de intervención psicológica empleada con deportistas debe precisar rigor metodológico y ético, en la línea de los trabajos de Cantón (2016) y de García-Naveira (2010).

En segundo lugar, se considera conveniente priorizar la intervención psicológica con los jugadores que acumulan una práctica de voleibol inferior a cinco años y de cinco a diez años. Dicha preferencia se debe a que éstos grupos están formados por deportistas con edades que oscilan entre los 13 y 21 años y en deportes de equipo parece ser el rango idóneo para favorecer el desarrollo de las habilidades psicológicas y afrontar la competición en las mejores condiciones (Roffé, 2016). En este sentido, nuestros resul- 
tados muestran la conveniencia de intervenir en estos dos grupos ya que se aprecian niveles más bajos de motivación y más altos de ansiedad que los registrados por el grupo de más edad que acumula diez o más años practicando voleibol. Por tanto, para el adecuado asesoramiento psicológico del deportista habría que contar con dicha variable temporal con el fin de mejorar el rendimiento y la motivación en el momento preciso.

En tercer lugar, para aumentar la motivación y reducir los niveles de ansiedad y agobio al decidir de jugadores de voleibol se precisa de actuaciones en al menos dos niveles: (1) intervención individual con los jugadores y (2) intervención grupal con el equipo. Esta organización de las actuaciones ya se han llevado a cabo con éxito en un trabajo previo de intervención con futbolistas (Olmedilla, Ortega, Andreu y Ortín, 2010). Además, se sabe que los programas que inciden sobre el control de la activación, la relajación y la visualización son positivamente valorados por parte de los deportistas (Olmedilla, Ortega, Boladeras, Ortín, Bazaco, 2013). Respecto a la intervención psicológica individual, se propone la puesta en marcha de ejercicios de respiración que permitan controlar el arousal fisiológico. Esta técnica ya ha sido usada con excelentes resultados en programas de preparación psicológica de deportistas (Abenza, González, Reyes, Reyes, Blas, Olmedilla, 2014). Además, también se plantea realizar entrenamiento de exposición en imaginación a diferentes situaciones conflictivas que pueden ocasionarse en el transcurso de la competición y que han resultado eficaces en otros trabajos (Bossio, Raimundi y Gómez, 2012). Para finalizar con la intervención individual, se recomienda el empleo de la técnica de reestructuración cognitiva de manera conjunta a la aplicación de las técnicas propuestas dado que la combinación de éstas ha mostrado mayor eficacia en la reducción de la ansiedad que el tratamiento cognitivo-conductual únicamente (Cano-Vindel, Dongil-Collado, Salguero y Wood, 2011). En relación con la intervención a nivel grupal, estudios previos ya han señalado la necesidad de realizar este tipo de intervenciones para mejora la motivación de los jugadores (García-Mas y Rivas-Garza, 2001). En la línea de éste trabajo, consideramos fundamental trabajar aspectos tales como la confianza, la cohesión del equipo, el clima de grupo, la concentración y el establecimiento de metas que van a mejorar al equipo. Así, se requiere de la figura del psicólogo deportivo integrada dentro de los clubes, tanto para la aplicación de estas técnicas como para la elaboración de programas de entrenamiento psicológico, que tengan en cuenta en su diseño la influencia de los años de experiencia de los jugadores y permitan intervenir en el momento preciso sobre los aspectos psicológicos del jugador.

\section{Referencias}

Abenza, L., González, J., Reyes, L., Blas, A., Olmedilla, A. (2014). Descripción y evaluación del entrenamiento psicológico de una deportista de regata clase laser radial. Revista Iberoamericana de Psicología del Ejercicio y el Deporte, 9 (1), 67-92.

Asociación Médica Mundial. (1964). Declaración de Helsinki de la Asociación Médica Mundial. Principios éticos para las investigaciones médicas en seres humanos. (64 Asamblea General, Fortaleza, 2013). Recuperado de http://www.wma.net/es/ 30publications/ 10policies/b3/

Ato, M., López, J. J. y Benavente, A. (2013). Un sistema de clasificación de los diseños de investigación en psicología. Anales de Psicología, 29, 1038-1059. http://dx.doi.org/10.6018/analesps.29.3.178511

Baena-Extremera, A. y Granero-Gallegos, A. (2015). Sexo y edad del alumnado sobre las orientaciones de meta en la Educación Física. Estudios Pedagógicos, 41(2), 25-39. http://dx.doi. org/10.4067/S0718-07052015000200002

Balaguer, I., Castillo, I. y Duda, J. L. (2003). La Escala de Motivación Deportiva: una nueva medida de la motivación intrínseca, motivación extrínseca y no-motivación. Análisis psicométrico de la versión española. En Actas de resúmenes del /l Congreso Internacional de Psicología aplicada al Deporte (p. 165). Madrid, España: Dykinson.

Balaguer, I., Castillo, I. y Duda, J. L. (2007). Propiedades psicométricas de la Escala de Motivación Deportiva en deportistas españoles. Revista Mexicana de Psicología, 24, 197- 207.

Boix, S. (2016). Efectos de la práctica del método Pilates sobre la salud psicosocial: Un estudio longitudinal (Tesis doctoral). Universidad Miguel Hernández de Elche, Alicante (España).

Bossio, M., Raimundi, M. J. y Gómez, L. (2012). Programa de entrenamiento en habilidades psicológicas en jugadoras de voleibol de alto rendimiento. Cuadernos de Psicología del Deporte, 12(1), 9-16.

Cano-Vindel, A., Dongil-Collado, E., Salguero, J. M. y Wood, C. (2011). Intervención cognitivo-conductual en los trastornos de ansiedad: una actualización. Informació Psicológica, 102, 4-27.

Cantón, E. (2016). La especialidad profesional en Psicología del Deporte. Revista de Psicología Aplicada al Deporte y al Ejercicio Físico, 1(e2), 1-12. https://dx.doi.org/10.5093/rpadef2016a2

Carratalá, E. (2004). Análisis de la teoría de las metas de logro y de la autodeterminación en los planes de especialización deportiva de la Generalitat Valenciana (Tesis doctoral). Universidad de Valencia, Valencia (España).

Catalá, P., Peñacoba, C., Velasco, L., Jareño, N. y Fernández-Serrano, A. (2016). La motivación como variable influyente en la ansiedad y autoconfianza de los deportistas. Informació Psicològica, 112, 14-28. http://dx.doi.org/10.14635/IPSIC.2016.112.2

Chamberlain, C. J. y Coelho, A. J. (1993). The perceptual side of action: decision-making in sport. Advances in Psychology, 102, 135-157.

Claver, F., Jiménez, R., del Villar, F., García-Mas, A. y Moreno, M. P. (2015). Motivación, conocimiento y toma de decisiones: Un estudio predictivo del éxito en voleibol. Revista de Psicología del Deporte, 24, 273-279.

Clancy, R. B., Herring, M. P., MacIntyre, T. E. y Campbell, M. J. (2016). A review of competitive sport motivation research. Psychology 
of Sport and Exercise, 27, 232-242. http://dx.doi.org/10.1016/j. psychsport.2016.09.003

Conejero, M., Claver, F., Fernández-Echevarría, C., González-Silva, J. y Moreno, M. P. (2017). Diseño y validación de un instrumento de observación para valorar la toma de decisiones en la acción de recepción en voleibol. Cultura, Ciencia y Deporte, 12, 6775. http://dx.doi.org/10.12800/ccd.v12i34.833

Deci, E. L. y Ryan, R. M. (1985). Intrinsic Motivation and Self-determination in Human Behavior. Nueva York, Estados Unidos: Plenum Press.

Estrada-Contreras, O. y Pérez-Córdoba, E. (2015). Investigaciones en Psicología del Deporte en la Universidad de Sevilla. En B. Sañudo, J. del Pozo y L. Carrasco (Eds.), La visión integradora de la investigación en Ciencias del Deporte (pp. 19-25). Sevilla, España: Grupo de investigación BIOFANEX, Universidad de Sevilla.

García-Mas, A. y Rivas-Garza, C. A. (2001). Veinte maneras de motivar a un equipo. Cuadernos de Psicología del Deporte, 1(1), 39-68.

García-Naveira, A. (2010). El psicólogo del deporte en el alto rendimiento: aportaciones y retos futuros. Papeles del Psicólogo, 31, 259-268.

García-Naveira, A. y Ruiz-Barquín, R. (2013). Diferencias de personalidad en entrenadores desde el modelo de Costa y McCrae. Cuadernos de Psicología del Deporte, 13(2), 53-62.

García, V., Ruiz, L. M. y Graupera, J. L. (2009). Perfiles decisionales de jugadores y jugadoras de voleibol de diferente nivel de pericia. Revista Internacional de Ciencias del Deporte, 14(5), 123-137.

Gil-Arias, A., Jiménez, R., Moreno, M. P., García-González, L., Moreno, B. y Del Villar, F. (2010). Análisis de la motivación intrínseca a través de las necesidades psicológicas básicas y la dimensión subjetiva de la toma de decisiones en jugadores de voleibol. Revista Iberoamericana de Psicología del Ejercicio y el Deporte, 5(1), 29-44.

Gil-Arias, A., Moreno, M. P., Claver, F., Moreno, A. y Del Villar, F. (2016). Manipulación de los condicionantes de la tarea en Educación Física: Una propuesta desde la pedagogía no lineal. Retos, 29, 22-27.

Hodges, N .J. Huys, R. y Starkes, J. L. (2007). Methodological review and evaluation of research in expert performance in sport. En G. Tenenbaum y R. C. Eklund (Eds.), Handbook of Sport Psychlology (pp.161-183). New Jersey, Estados Unidos: John Wiley \& Sons.

Irwin, B. C. y Feltz, D. L. (2016). Motivation gains in sport and exercise groups. En R. J. Schinke, K. R. McGannon, B. Smith, R. J. Schinke, K. R. McGannon y B. Smith (Eds.), Routledge international handbook of sport psychology (pp. 494-504). New York: Routledge/Taylor \& Francis Group.

Iso-Ahola, S. E. y St.Clair, B. (2000). Toward a theory of exercise motivation. Quest, 52, 131-147.

Jiménez, C. (2007). Análisis de la toma de decisiones en los deportes colectivos: Estrategias de los jugadores aleros de baloncesto en posesión de balón. Sevilla, España: Wanceulen.

Jiménez, M. I. y López-Zafra, E. (2009). Inteligencia emocional y rendimiento escolar: Estado actual de la cuestión. Revista Latinoamericana de Psicología, 47(1), 67-77.

Jõesaar, H., Hein, V. y Hagger, M. S. (2011). Youth athletes' perception of autonomy support from the coach, peer motivational climate and intrinsic motivation in sport setting: One-year effects. Psychology of Sport and Exercise, 13, 257-262. https:// doi.org/10.1016/i.psychsport.2011.12.001
Johnson, J. G. (2006). Cognitive modeling of decision making in sports. Psychology of Sport and Exercise, 7, 631-652.

Konzag, I. (1992). Actividad cognitiva y formación del jugador. Revista de Entrenamiento Deportivo, 6(6), 35-44.

Lorenzo, J., Gómez, M. A., Pujals, C. y Lorenzo, A. (2012). Análisis de los efectos de un programa de intervención psicológica en jóvenes jugadores de baloncesto. Revista de Psicología del Deporte $21,43-48$.

Moreno, J. A. y Martínez, A. (2006). Importancia de la Teoría de la Autodeterminación en la práctica físico-deportiva: Fundamentos e implicaciones prácticas. Cuadernos de Psicología del Deporte, 6(2), 39-54.

Núñez, J. L., Martín-Albo, J. y Navarro, J. G. (2007). Propiedades psicométricas de la versión española de la Escala de Motivación Deportiva. Revista de Psicología del Deporte, 16, 211-223.

Olmedilla, A., Ortega, E. Andreu, M. D. y Ortín, F. (2010). Programa de intervención psicológica en futbolistas: evaluación de habilidades psicológicas mediante el CPRD. Revista de Psicología del Deporte, 19, 249-262.

Olmedilla, A., Ortega, E., Boladeras, A., Ortín Montero, F., Bazaco, M. J. (2013). Entrenamiento en estrategias y técnicas psicológicas y percepción de ayuda en futbolistas juveniles. SPORT TK-Revista EuroAmericana de Ciencias del Deporte, 2(1), 51-58.

Palmero, F. (2005). Motivación: Conducta y Proceso. Revista Electrónica de Motivación y Emoción, 8(20-21), 1-29.

Pelletier, L. G., Fortier, M. S., Vallerand, R. J., Tuson, K. M., Briere, N. M. y Blais, M. R. (1995). Toward a new measure of intrinsic motivation, extrinsic motivation, and amotivation in sports: The sport motivation scale (SMS). Journal of Sport and Exercise Psychology, 17, 35-53.

Reche, C., Cepero, M. y Rojas, F. J. (2010). Consideraciones en el entrenamiento de habilidades psicológicas en esgrima. Cuadernos de Psicología del Deporte, 13(2), 83-88.

Roberts, G. C. (2001). Understanding the dynamics of motivation in physical activity: The influence of achievement goals on motivation processes. En G. C. Roberts (Ed.), Advances in motivation in sport and exercise (pp. 1-50). Champaign, Estados Unidos: Human Kinetics.

Roffé, M. (2016). La preparación psicológica de la Selección Nacional Absoluta de Colombia para el Mundial de Fútbol Brasil 2014. Revista de Psicología Aplicada al Deporte y al Ejercicio Físico, 1, e3. https://doi.org/10.5093/rpadef2016a3

Ruiz, L. M. y Graupera, J. L. (2005). Dimensión subjetiva de la toma de decisiones en el de- porte: Desarrollo y validación del cuestionario CETD de estilo de decisión en el deporte. Motricidad. European Journal of Human Movement, 14, 95-107.

Ruiz, L. M., Graupera, J. L. y Navarro, F. (2000). Construcción, análisis psicométrico y tipificación de un cuestionario de decisión en el deporte. Madrid, España: Centro de Alto Rendimiento y de Investigaciones en el Deporte, Consejo Superior de Deportes.

Ruiz, L. M., Graupera, J. L., Arruza, J. A., Mendoza, N., Sánchez, F. y Del Río, P. (2002). Validación transcultural de Cuestionario CETD de Estilos de Toma de Decisión en el deporte. Madrid, España: Consejo Superior de Deportes.

Ryan, R. M. y Deci, E. L. (2000). Self-determination theory and the facilitation of intrinsic motivation, social development and weIl-being. American Psychologist, 55, 68-78. 
Standage, M. y Vallerand, R. J. (2014). Motivation in sport and exercise groups: A self-determination theory perspective. En M. Beauchamp y M. Eys, (Eds.), Group dynamics in exercise and sport psychology (pp. 259-278). NuevaYork, Estados Unidos: Routledge.

Thomas, J. R., French, K. E. y Humphries, C. A. (1986). Knowledge development and sport performance: Directions for motor behaviour research. Journal of Sport Psychology, 8, 259-272.
Unisport. (1992). Carta Europea del Deporte. Málaga, España: Autor. Zeelenberg, M., Nelissen, R. M. A. y Pieters, R. (2008). Emotion, motivation, decision making: A feeling-is-for-doing approach. En H. Plessner, C. Betsch y T. Betsch (Eds.), Intuition in judgment and decision making (pp. 173-190). Mahwah,NJ, Estados Unidos: Erlbaum. 